\title{
Long-term treatment outcomes of children and adolescents with lymphoblastic lymphoma treated with various regimens: a single-center analysis
}

\author{
Ho Jung Choi ${ }^{1}$, Juhee Shin ${ }^{1,2}$, Sunghan Kang ${ }^{1,2}$, Jin Kyung Suh ${ }^{1,2}$, Hyery Kim ${ }^{1,2}$, Kyung-Nam Koh ${ }^{1,2}$, \\ Ho Joon $\operatorname{Im}^{1,2}$ \\ ${ }^{1}$ Department of Pediatrics, University of Ulsan College of Medicine, Asan Medical Center, ${ }^{2}$ Division of Pediatric \\ Hematology/Oncology, Department of Pediatrics, University of Ulsan College of Medicine, Asan Medical Center, Seoul, Korea
}

p-ISSN 2287-979X / e-ISSN 2288-0011 https://doi.org/10.5045/br.2020.2020220 Blood Res 2020;55:262-274.

Received on August 29, 2020 Revised on November 2, 2020 Accepted on November 12, 2020
*This study was supported by a grant from National Research Foundation of Korea (NRF) grant funded by the Korean government (MSIT) (No. NRF-2018R1C1 B5047092).

\section{Correspondence to}

Hyery Kim, M.D., Ph.D.

Division of Pediatric Hematology/

Oncology, Department of Pediatrics,

University of Ulsan College of Medicine,

Asan Medical Center, 88-1 Olympic-ro

43-gil, Songpa-gu, Seoul 05505, Korea

E-mail: taban@hanmail.net

(C) 2020 Korean Society of Hematology

\section{Background}

Lymphoblastic lymphoma ( $\mathrm{LBL})$ is the second most common subtype of pediatric non-Hodgkin lymphoma. Modified treatments derived from the LSA2-L2 regimen resulted in encouraging survival, but toxicities and long-term sequelae have been problematic. At present, the acute lymphoblastic leukemia (ALL)-type protocol has demonstrated efficacy in LBL. We analyzed the outcomes of children and adolescents with LBL treated with various regimens.

\section{Methods}

From 1991-2018, this study enrolled 63 patients diagnosed with LBL at Asan Medical Center. Medical records were retrospectively analyzed.

\section{Results}

Among 63 patients, most patients (38.1\%) presented with stage IV at diagnosis, and two had central nervous system (CNS) involvement. At a median follow-up of 160 months, the 5 -year event free survival (EFS), overall survival (OS), and relapse free survival (RFS) were $68.8 \%, 79.3 \%$, and $71.3 \%$, respectively. Among 61 patients who received chemotherapy, 27 patients (44.3\%) received the NY protocol, and $14(23.0 \%)$ received the ALL-type protocol. There was no significant difference in 5-yr OS (85.2\%/78.6\%), EFS (73.5\%/78.6\%), and RFS (73.5\%/78.6\%) between the NY and ALL protocol groups, regardless of immunophenotype. Thirteen patients (21.3\%) received prophylactic cranial radiotherapy with no difference in the incidence of CNS relapse based on irradiation.

\section{Conclusion}

This study showed no difference in outcome between the NY and ALL-type protocols, regardless of stage or immunophenotype. In addition to improving the effectiveness of treatment, it is necessary to continuously appraise the appropriate chemotherapy regimen, considering toxicities and long-term prognosis, for pediatric LBL.

Key Words Lymphoblastic lymphoma, Child, Survival, Chemotherapy, New York protocol

\section{INTRODUCTION}

Lymphoblastic lymphoma (LBL) is the second most common subtype of pediatric non-Hodgkin lymphoma (NHL). Through the development of an intensified regimen applied according to stage, histology, or immunophenotype, the outcome of pediatric LBL treatment has been improving for the past 30 years [1].

In the past, the Children's Cancer Group (CCG) conducted separate treatments for LBL and lymphomatous ALL. In 1988, two chemotherapeutic strategies used for NHL, the COMP (4-drug regimen, cyclophosphamide, vincristine, methotrexate, and prednisolone) and LSA2-L2 (10-drug regimen, cyclophosphamide, vincristine, prednisone, daunomycin, methotrexate, cytarabine, thioguanine, asparaginase, and carmus- 
tine) were compared, and the superiority of the LSA2-L2 protocol was demonstrated in LBL, especially in disseminated disease [2, 3].

The New York (NY) protocol, which was primarily developed for ALL, is a modified version of the LSA2-L2 protocol. This regimen is characterized by rapid intensive induction followed by alternating non-cross-resistant combination chemotherapy. Because the LSA2-L2 protocol shows a superior outcome over the 4-drug regimen (COMP) in disseminated lymphoma or LBL [2], the NY protocol has been used for the treatment of pediatric LBL [4]. This protocol resulted in encouraging survival, but toxicities and long-term sequelae incurred by intensive chemotherapy have been problematic $[5,6]$. Therefore, reducing the risk of treatment-related toxicities has been discussed in the treatment of pediatric LBL [7].

International study groups, including the Berlin-FrankfurtMünster regimen (BFM) have used reduced doses of anthracyclines and cyclophosphamide for pediatric NHL, which do not compromise survival outcomes [8]. Likewise, prophylactic cranial radiotherapy (PCRT) is no longer included in the treatment regimen for LBL because it shows no additional effect in reducing CNS relapses and might cause additional neurologic toxicity [9-14]. For CNS-negative patients with stage III or IV LBL and sufficient response to induction chemotherapy, treatment without PCRT is non-inferior to treatment with PCRT [15].

LBL is classified as a high-grade lymphoid malignancy according to the World Health Organization (WHO) classification, and when there is greater than $25 \%$ marrow replacement by lymphoblasts, the designation of ALL is given [16]. Thus, disseminated LBL has been treated with an ALL-type protocol. Previous studies have shown improved long-term outcomes with the ALL-type protocol in LBL $[1,3,9,17,18]$. In particular, changing to the ALL-type protocol has allowed replacing PCRT with intensified intrathecal methotrexate and high-dose methotrexate (HD-MTX).

This report analyzed the outcomes of children and adolescents with LBL treated with various regimens, and outcome differences according to PCRT, at a single center.

\section{MATERIALS AND METHODS}

\section{Patients}

This study enrolled children and adolescents younger than 20 years who were diagnosed with LBL and treated at Asan Medical Center Children's Hospital between December 1991 and March 2018. The last follow-up of the study was conducted in April 2020. Data were reviewed retrospectively. The diagnosis of LBL was distinguished from ALL based on a bone marrow involvement of $<25 \%$, and with the analysis of cerebrospinal fluid to evaluate initial CNS involvement. Extramedullary involvement was evaluated using computed tomography (CT) or magnetic resonance imaging (MRI). This study was approved by the Institutional Review Board of the Asan Medical Center (IRB No. 2020-1001).

\section{Methods}

Various chemotherapy regimens were administered to the patients in this study. In the 1990s, the addition of daunorubicin and asparaginase to the basic COMP protocol (ADCOMP), LSA2-L2, or the Pediatric Oncology Group (POG) regimens, such as POG-9219, POG-9317, POG-9404, or POG-9406, were mainly used. After the 2000s, the ALL-type protocol (Children's Cancer Group-1882, Korean multicenter high-risk ALL protocol [19], or Children's Oncology GroupA5971 [9]) was applied to B-cell type LBL, while a modified NY protocol (CCG-106B or CCG-1901) (Supplementary Table 1) was mainly applied to T-lymphoblastic lymphoma (T-LBL).

The modified Children's Cancer Group (CCG)-1882 regimen, an example of an ALL-type protocol, is a regimen that includes two interim maintenance schedules and delayed intensifications after induction and consolidation (Supplementary Table 1). Representative examples of the NY protocol are CCG-1901 or CCG-106B, which are two-year regimens that repeat maintenance every three months after intensive induction and consolidation. Treatment outcomes were compared by dividing patients into a NY protocol group and an ALL-type protocol group. PCRT was mainly applied by incorporation into the NY protocol or in patients with advanced stages prior to 2013 . Radiation was administered to extramedullary lesions when there was a partial response after chemotherapy, especially to large mediastinal masses. For patients who relapsed after chemotherapy, protocols such as ifosfamide, carboplatin, and etoposide (ICE) [20], or dexamethasone, etoposide, cisplatin, cytarabine, and L-asparaginase (DECAL) [21] were used as salvage treatments. Stem cell transplantation was performed in poor responders who had advanced disease at the time of diagnosis or in patients with relapsed disease.

\section{Statistical analysis}

In this study, the CCG-1882 and the Korean multicenter high-risk ALL protocol (CALL-1501) were collectively called the "ALL-type protocol", while the CCG-106B and CCG-1901 were collectively termed as the "NY protocol", for statistical analysis.

Event-free survival (EFS) was calculated from the time of diagnosis to the first occurrence of progression, relapse, death, or date last seen for patients who remained alive and disease-free. The events considered were relapse at any site, death in remission, or a second malignant neoplasm, whichever occurred first. Relapse-free survival (RFS) was defined as the date from diagnosis to relapse. Overall survival (OS) was defined as the date from diagnosis to death from any cause, or date last seen for patients who remained alive. Survival rates were estimated using the Kaplan-Meier method. The log-rank test was used to compare the estimated survival distributions by patient characteristics. All analyses were performed using SPSS version 18 (SPSS Inc., Chicago, IL, USA), and statistical significance was accepted for $P<0.05$. 


\section{RESULTS}

\section{Patient characteristics}

From December 1991 to March 2018, 63 patients were

Table 1. Characteristics of patients.

\begin{tabular}{lc}
\hline \multicolumn{1}{c}{ Characteristics } & $\mathrm{N}(\%)$ \\
\hline Sex & \\
Male & $34(54.0 \%)$ \\
Female & $29(46.0 \%)$ \\
Median age at diagnosis (range, yr) & $8(2-19)$ \\
Immunophenotype & \\
T-LBL & $39(61.9 \%)$ \\
B-LBL & $21(33.3 \%)$ \\
Unknown & $3(4.8 \%)$ \\
Mediastinal mass & $29(46.0 \%)$ \\
Median LDH level (IU/L) & $962(447-3,297)$ \\
Stage ${ }^{a}$ & \\
I & $8(12.7 \%)$ \\
II & $10(15.9 \%)$ \\
III & $21(33.3 \%)$ \\
IV & $24(38.1 \%)$ \\
Initial CNS involvement & \\
Involved (+) & $2(3.2 \%)$ \\
Involved (-) & $59(93.7 \%)$ \\
Unknown & $2(3.2 \%)$ \\
Initial BM involvement & \\
Involved (+) & $21(33.3 \%)$ \\
Involved (-) & $42(66.7 \%)$ \\
\hline
\end{tabular}

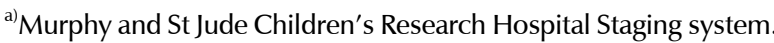
Abbreviations: $\mathrm{BM}$, bone marrow; CNS, central nervous system; $\mathrm{LBL}$, lymphoblastic lymphoma; LDH, lactate dehydrogenase. included in this study (Table 1). The median age at diagnosis was 8 years (range, 2-19 yr). T-LBL was the most common immunophenotype at diagnosis (39 patients, 61.9\%), and stage IV was the most common stage at diagnosis (24 patients, $38.1 \%$ ). There were 45 advanced stage (stage III/IV) patients and 18 early stage (stage $\mathrm{I} / \mathrm{II}$ ) patients. A mediastinal mass was confirmed with CT scans in 29 patients $(46.0 \%)$ with T-LBL, which comprised $86.2 \%$ (25 patients) of T-LBL cases. Data were analyzed as of April 30, 2020.

\section{Treatment}

Among 63 children, 2 patients died of gastrointestinal (GI) bleeding before the start of chemotherapy, and 61 patients underwent initial chemotherapy (Table 2). The NY protocol was the most common protocol used (27 patients, $44.3 \%$ ), followed by the ALL-type protocol (14 patients, 23.0\%), POG regimens (7 patients, 11.5\%), LSA2-L2 (7 patients, $11.5 \%$ ), and AD-COMP (6 patients, 9.8\%). There was no statistically significant difference in age, sex, immunophenotypes, stages, initial CNS or BM involvement between the NY protocol group and ALL-type protocol group (Supplementary Table 2).

A total of 21 patients were treated with therapeutic radiotherapy on primary (16 patients) or relapsed disease ( 5 patients). The chest or mediastinum (13 patients) was the most common site, followed by the craniospinal area (6 patients), neck ( 3 patients), and the inguinal area (1 patient). The median radiation dose was $1,800 \mathrm{cGy}$ (range, 50-5,580 cGy). None of the 6 patients who underwent therapeutic irradiation of the craniospinal area had prior PCRT; 2 had relapsed lymphoma in the CNS, and the other 4 had disease involvement of the forehead, periauricular area, orbit, or the spine.

Table 2. Number of patients according to chemotherapy protocol.

\begin{tabular}{|c|c|c|c|c|c|c|}
\hline \multicolumn{2}{|c|}{ Stage } & \multirow{3}{*}{$\begin{array}{c}\text { AD-COMP } \\
1\end{array}$} & \multirow{3}{*}{$\begin{array}{c}\text { ALL-type regimen }^{\text {a) }} \\
3 \\
3\end{array}$} & \multirow{3}{*}{$\begin{array}{c}\text { LSA2-L2 }^{\text {b) }} \\
\text { N } \\
0\end{array}$} & \multirow{3}{*}{$\begin{array}{l}\text { NY } \\
\text { regimen } \\
2 \\
\end{array}$} & \multirow{3}{*}{$\begin{array}{c}\text { POG regimen }^{\mathrm{c})} \\
1\end{array}$} \\
\hline & & & & & & \\
\hline \multirow[t]{5}{*}{ B-LBL } & I & & & & & \\
\hline & II & 0 & 1 & 0 & 1 & 2 \\
\hline & III & 0 & 0 & 0 & 1 & 1 \\
\hline & IV & 1 & 2 & 1 & 3 & 1 \\
\hline & Total & 2 & 6 & 1 & 7 & 5 \\
\hline \multirow[t]{4}{*}{ T-LBL } & II & 0 & 1 & 0 & 4 & 1 \\
\hline & III & 2 & 5 & 3 & 8 & 0 \\
\hline & IV & 1 & 2 & 2 & 7 & 1 \\
\hline & Total & 3 & 8 & 5 & 19 & 2 \\
\hline \multirow[t]{3}{*}{ Unknown } & I & 1 & 0 & 0 & 0 & 0 \\
\hline & III & 0 & 0 & 0 & 1 & 0 \\
\hline & IV & 0 & 0 & 1 & 0 & 0 \\
\hline Total & & $6(9.8 \%)$ & $14(23.0 \%)$ & 7 (11.5\%) & 27 (44.3\%) & 7 (11.5\%) \\
\hline
\end{tabular}

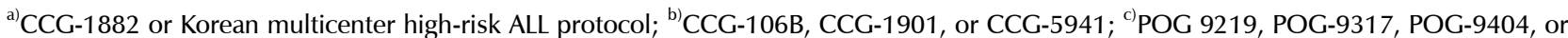
POG-9406. There was no statistically significant difference in age, sex, immunophenotypes, stages, initial CNS or BM involvement between patients of the NY protocol group, and those in the ALL-type protocol group.

Abbreviations: AD-COMP, addition of daunorubicin and asparaginase to the basic COMP protocol; ALL, acute lymphoblastic leukemia; LBL, lymphoblastic lymphoma; NY, New York; POG, Pediatric Oncology Group. 


\section{Overall outcome}

The median follow-up of all patients was 160 months (range, 0-340 mo). During the follow-up period, the 5-year OS of the 63 patients was $79.3 \pm 5.1 \%$, the 5-year EFS was $68.8 \pm 6.6 \%$, and the RFS was $71.3 \pm 5.7 \%$ (Fig. 1).

When analyzed by the initial stage, there was a tendency for a lower OS or EFS in the advanced stages, but there was no statistical difference (5-year OS, $P=0.325$; EFS, $P=0.884$ ) (Fig. 2). The RFS of stage I, II, III, and IV patients was $75.0 \pm 15.3 \%, 88.9 \pm 10.5 \%, 75.0 \pm 9.7 \%$, and $76.3 \pm 9.3 \%$, respectively $(P=0.818)$. The RFS of stage I patients was relatively low; however, all relapsed patients with initial stage I disease succeeded with salvage treatment.

When comparing survival rates in advanced stage patients by immunophenotype, the 5-year OS was $70.0 \%$ for patients with B-LBL and $72.6 \%$ for patients with T-LBL, without statistical difference $(P=0.907)$ (Fig. 3$)$. The EFS was $56.0 \%$ and $69.4 \%$ for patients with $\mathrm{B}-$ and T-LBL, respectively $(P=0.801)$.

There were 18 events: 2 early deaths due to bleeding of the involved GI tract, 13 relapses, and 3 deaths without relapse. Six patients had B-LBL, 11 patients had T-LBL, and $1 \mathrm{had}$ an unknown immunophenotype at diagnosis. Fourteen events occurred in patients with stage III/IV disease, and 4 events occurred in those with stage I/II disease. Among the 18 patients with events, 7 patients were treated with the NY protocol (6 with CCG-1901 and 1 with CCG-5941), three with the ALL-type protocol (2 with CCG-1882 and 1 with COG-A5971), 2 with AD-COMP, 2 with the POG regimen (1 with POG-9317 and 1 with POG-9404), and 2 with LSA2-L2. Two patients died of GI bleeding before chemotherapy (Fig. 4).

A total of 13 patients died; 2 patients who failed to start chemotherapy due to deterioration of vital signs died before chemotherapy or during treatment with dexamethasone alone due to bleeding of the GI tract, 2 died of septic shock during induction, 1 patient with relapsed T-LBL died of acute renal failure during chemotherapy with the ICE protocol, and 8 died from progressive lymphoma. All 3 patients who died of treatment-related complications were treated

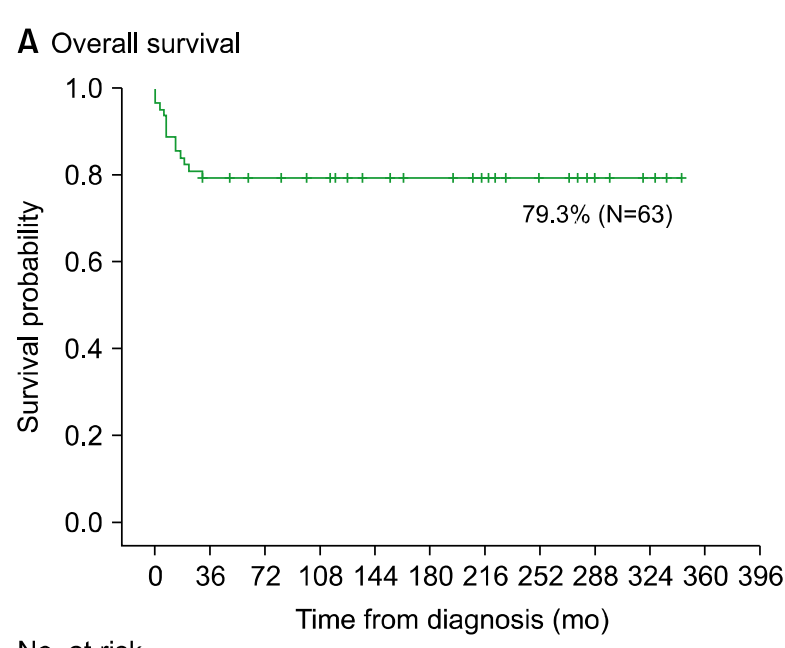

No. at risk

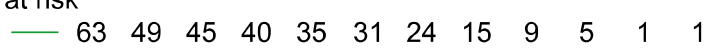

C Relapse free survival

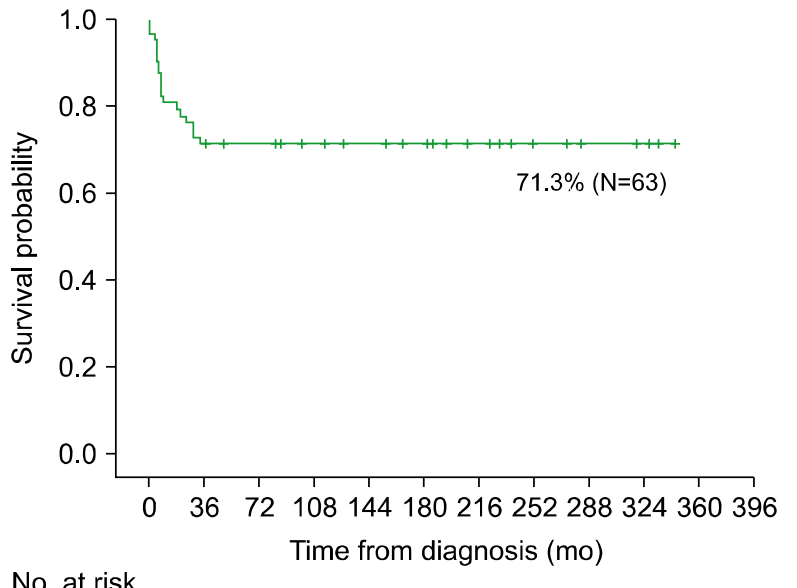

No. at risk

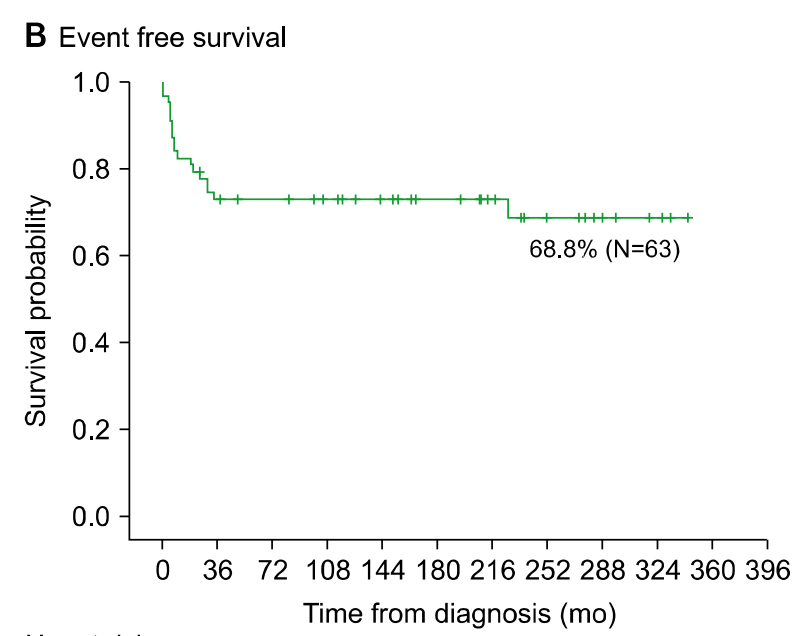

No. at risk

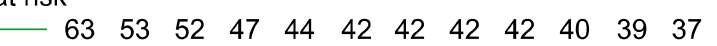

Fig. 1. Survival outcomes of patients. The overall survival (A), event-free survival (B), and relapse-free survival rates (C) were $79.3 \pm 5.1 \%, 68.8 \pm 6.6 \%$, and $71.3 \pm 5.7 \%$, respectively. 


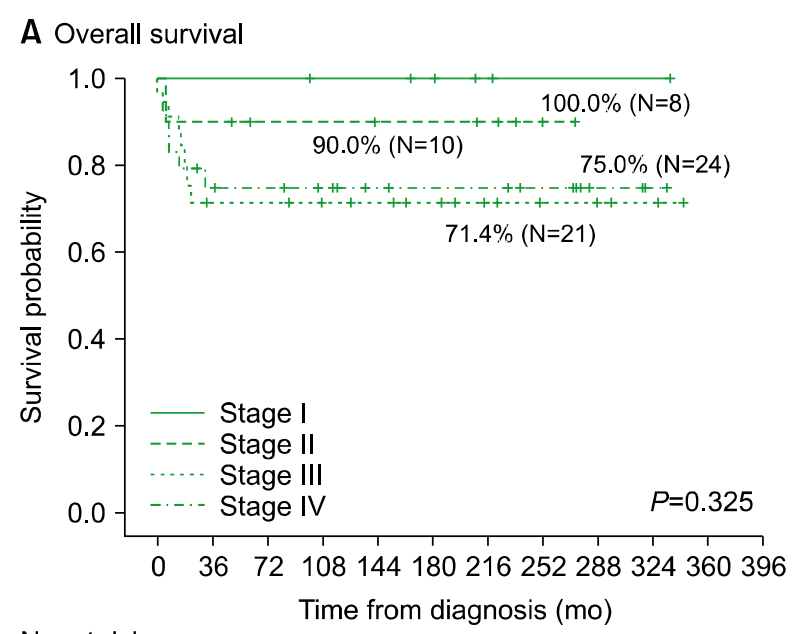

No. at risk

$\begin{array}{ccccccccccccc}- & 8 & 8 & 8 & 8 & 8 & 8 & 8 & 8 & 8 & 8 & 8 & 8 \\ --- & 10 & 10 & 7 & 7 & 6 & 6 & 5 & 3 & 1 & 1 & 1 & 1 \\ \cdots \cdots & 21 & 15 & 15 & 13 & 12 & 10 & 7 & 5 & 5 & 3 & 1 & 1 \\ -.- & 24 & 18 & 17 & 15 & 12 & 11 & 11 & 8 & 4 & 2 & 1 & 1\end{array}$

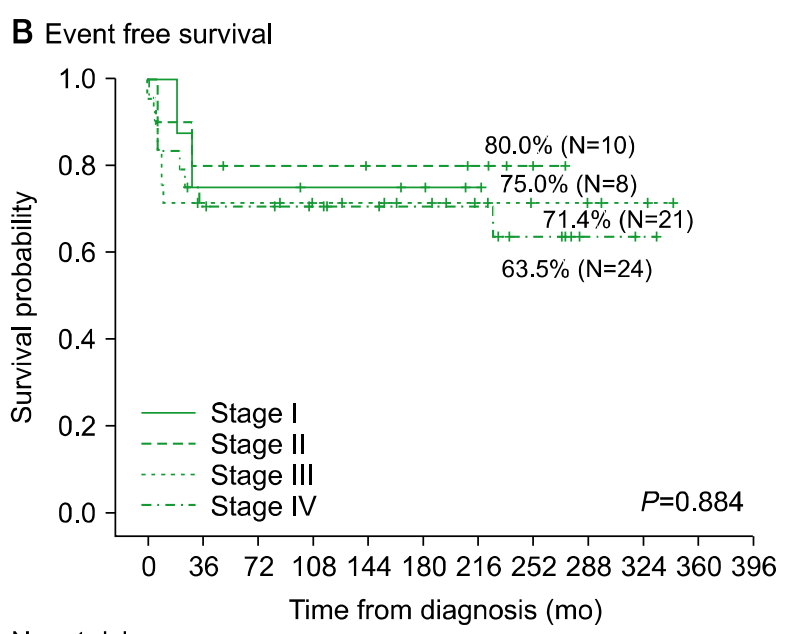

No. at risk

$\begin{array}{ccccccccccccc} & 8 & 7 & 7 & 6 & 6 & 5 & 2 & 1 & 1 & 1 & 1 & 1 \\ -\cdots & 10 & 9 & 7 & 7 & 6 & 6 & 5 & 3 & 1 & 1 & 1 & 1 \\ \cdots \cdots & 21 & 15 & 15 & 13 & 12 & 10 & 7 & 5 & 5 & 3 & 1 & 1 \\ \ldots-\cdots & 24 & 17 & 16 & 14 & 12 & 11 & 11 & 7 & 3 & 2 & 1 & 1\end{array}$

Fig. 2. When analyzed according to initial stage, patients with stage III/IV disease show lower survival rates than others. However the overall survival (A), and event-free survival rates (B) were not statistically different.

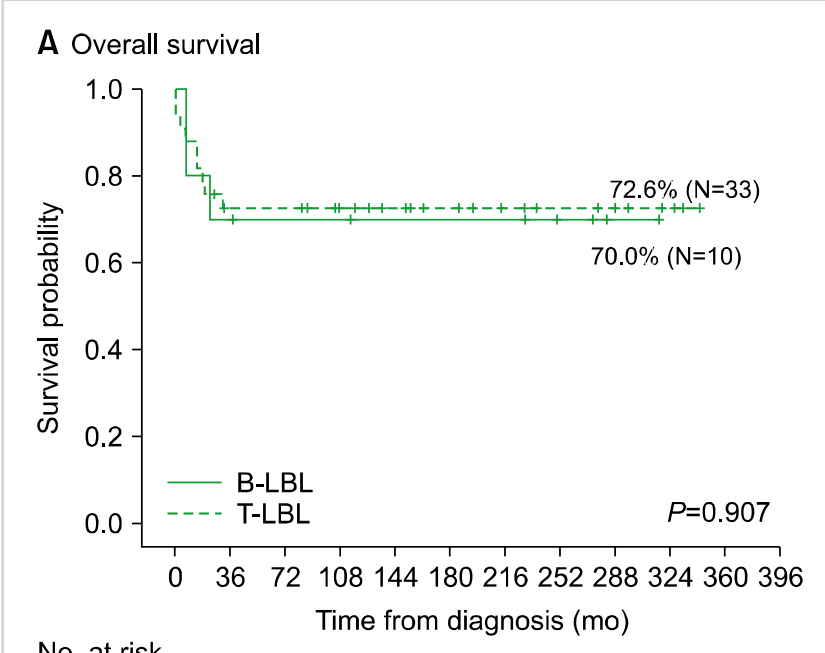

No. at risk

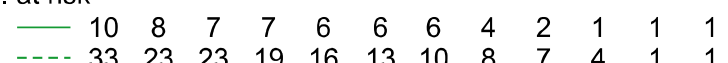

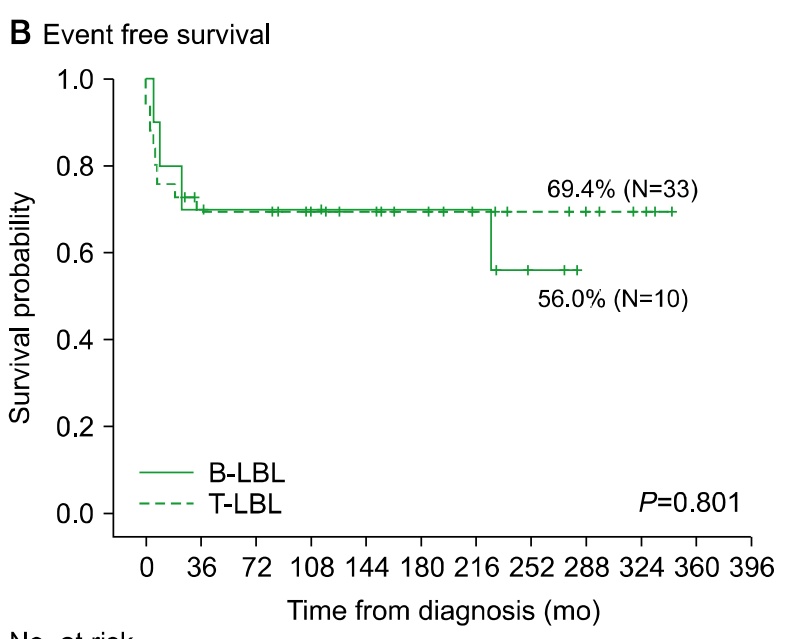

No. at risk

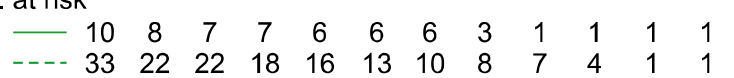

Fig. 3. When advanced stage patients were analyzed according to immunophenotype, the overall survival (A) and event-free survival rates (B) were not statistically different between B- and T-LBL $(\mathrm{N}=43)$.

with the ALL-type protocol (Fig. 4).

Among the 13 patients who died, 4 patients were treated with the NY protocol (3 with CCG-1901 and 1 with CCG-5941), 3 with the ALL-type protocol (2 with CCG-1882 and 1 with COG-A5971), 2 with LSA2-L2, and 2 with the POG regimen (1 with POG-9404 and 1 with POG-9317).

Three patients underwent stem cell transplantation (Table 3). Two patients underwent autologous peripheral blood stem cell transplantation (PBSCT) after relapse. A 14-year-old patient with B-LBL (Patient 1) was initially treated with
POG-9404; however, the mediastinal mass increased, and a new lesion in the kidney was detected after consolidation. After relapse, salvage chemotherapy with POG-9406, autologous PBSCT, and mediastinal irradiation were performed. However, a second relapse occurred in the brain, and the patient succumbed to relapsed lymphoma.

A 7-year-old patient (Patient 2) with initial stage IV T-LBL relapsed in the mediastinum during treatment with the NY protocol. After relapse, salvage chemotherapy was performed using the ICE protocol, and autologous PBSCT was performed 


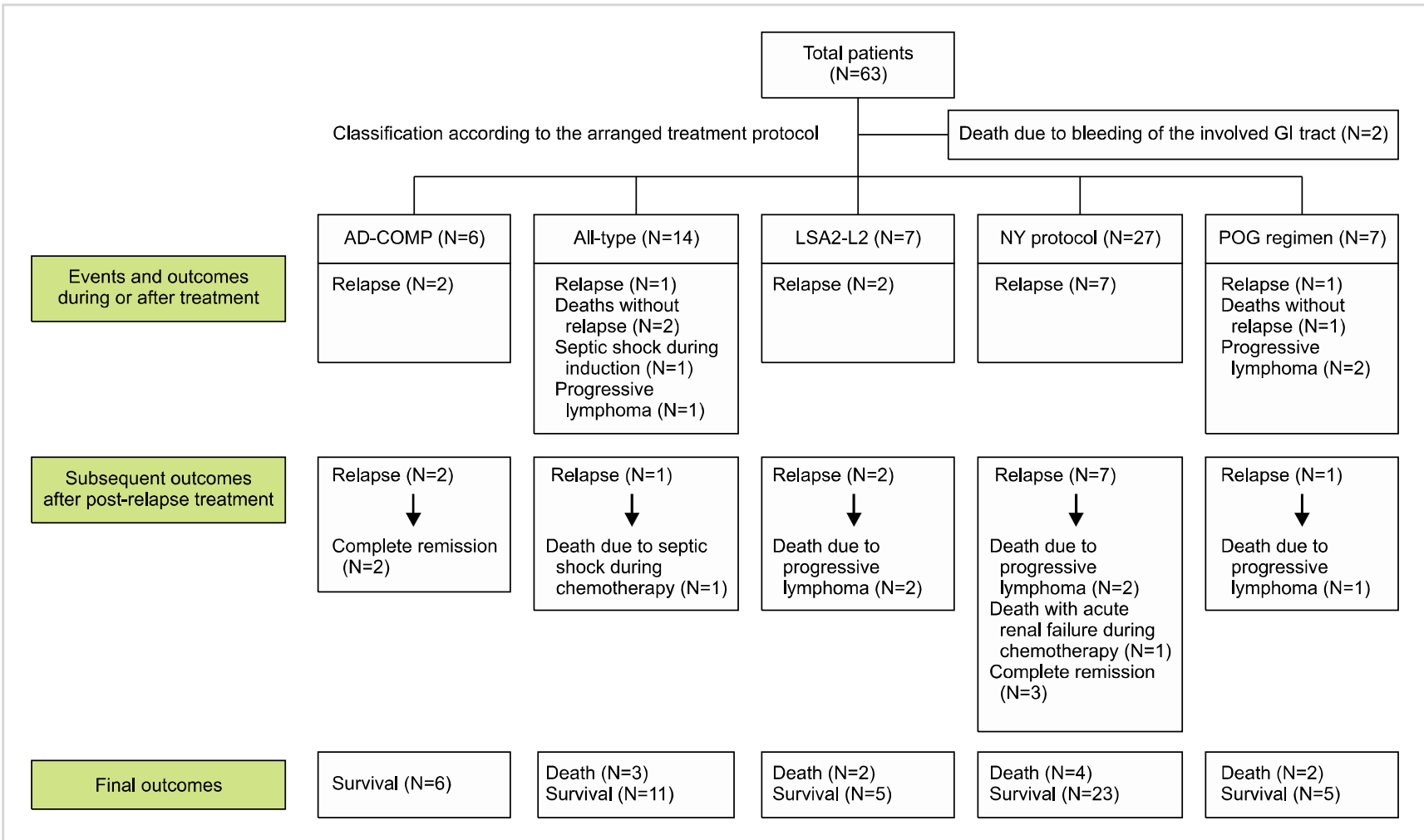

Fig. 4. There were 18 events, and 13 patients died. All patients alive to date are disease-free.

Table 3. Patients who received stem cell transplantation.

\begin{tabular}{|c|c|c|c|}
\hline & Patient 1 & Patient 2 & Patient 3 \\
\hline Age at diagnosis $(\mathrm{yr}) / \mathrm{sex}$ & $14 / F$ & $7 / \mathrm{M}$ & $8 / \mathrm{M}$ \\
\hline Immunophenotype & B-cell & T-cell & T-cell \\
\hline Stage & III & II & IV \\
\hline CNS involvement & None & None & None \\
\hline Initial Chemotherapy & POG-9404 & COG-1901 & COG-1901 \\
\hline Reason of transplantation & Relapse (mediastinum, kidney) & Relapse (mediastinum) & $\begin{array}{l}\text { Initial extensive stage (BM, } \\
\text { mediastinum), poor response }\end{array}$ \\
\hline Treatment after relapse & $\begin{array}{l}\text { POG-9406, mediastinal } \\
\text { radiotherapy }\end{array}$ & ICE protocol & - \\
\hline Type of transplantation & Autologous PBSCT & Autologous PBSCT & Matched sibling donor PBSCT \\
\hline Conditioning regimen & TBI/Cy/VP16 & $\mathrm{Bu} / \mathrm{Cy} / \mathrm{VP} 16$ & TBI/Cy/rATG \\
\hline Local radiotherapy & Mediastinum (post-PBSCT) & PCRT mediastinum (post-PBSCT) & - \\
\hline Current status & $\begin{array}{l}\text { Death due to relapsed disease } \\
\text { (post-PBSCT } 4 \mathrm{mo} \text { ) }\end{array}$ & Alive without disease ( 1 yr 5 mo) & Alive without disease (7 mo) \\
\hline
\end{tabular}

at complete remission status. He is alive, without disease, 17 months after PBSCT.

An 8-year old patient with T-LBL (Patient 3) had a large mediastinal mass and bone marrow involvement at diagnosis. Residual bone marrow disease was identified after induction chemotherapy, and residual mediastinal lymphoma activity was identified by positron emission tomography-CT after the first maintenance cycle. Allogeneic PBSCT with a match- ed sibling donor was planned instead of autologous PBSCT because of the initial bone marrow involvement. The patient is alive, without disease, 7 months after PBSCT.

During the follow-up period, several long-term complications were observed. In a 4-year-old patient who underwent initial chemotherapy with AD-COMP for T-LBL, radiotherapy was performed for a large mass of the chest. Subsequently, he developed radiotherapy-induced pneumo- 

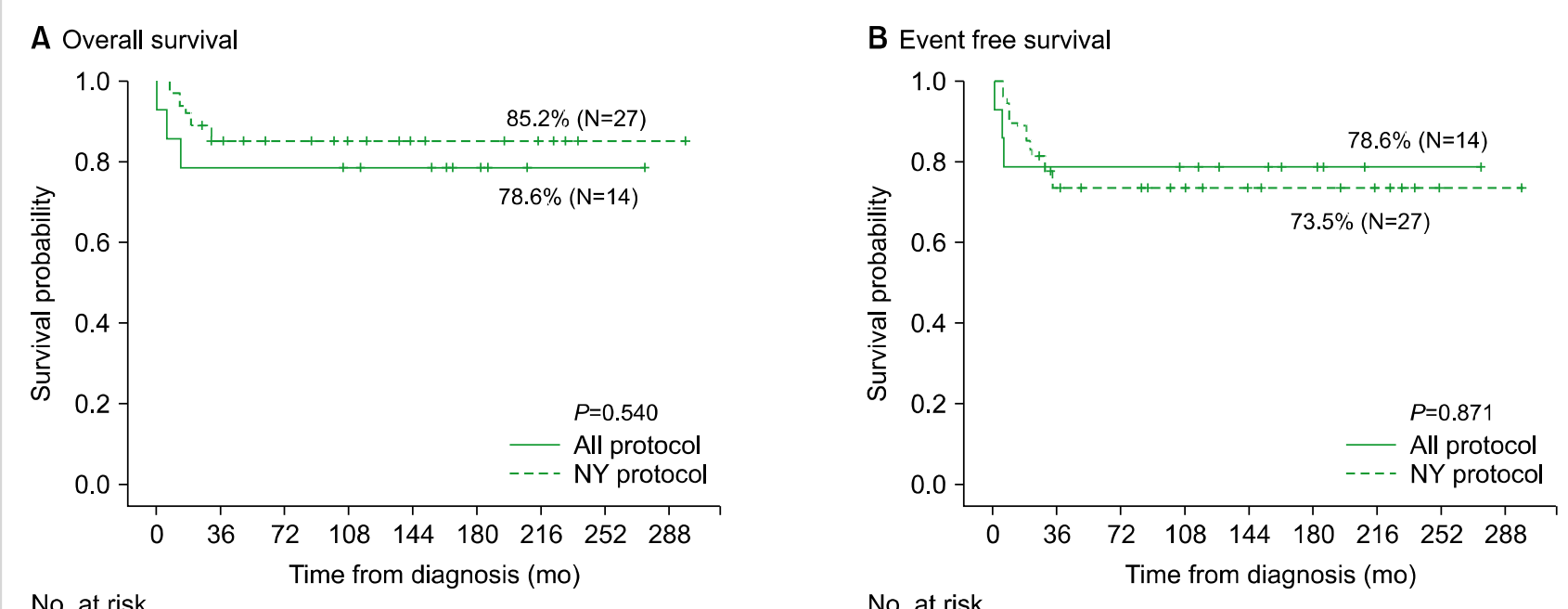
o. at risk
$\begin{array}{lllllllll}14 & 12 & 12 & 11 & 9 & 6 & 2 & 2 & 1\end{array}$

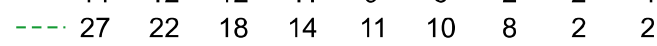

No. at risk

$\begin{array}{rrrrcrrrr}-14 & 12 & 12 & 11 & 9 & 6 & 2 & 2 & 1 \\ --\cdot 27 & 19 & 16 & 12 & 10 & 9 & 7 & 2 & 2\end{array}$

C Relapse free survival

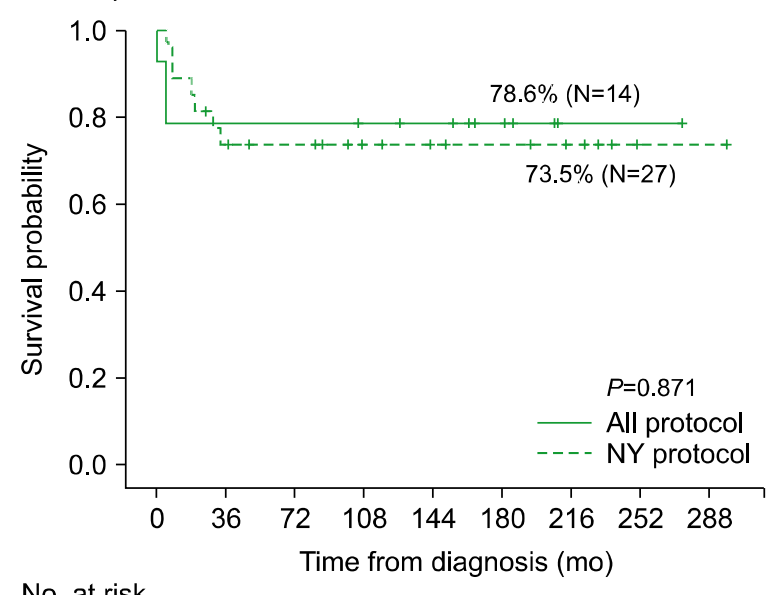

No. at risk

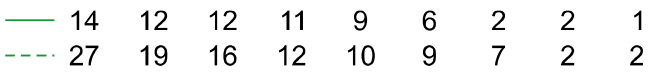

Fig. 5. Treatment outcome according to initial chemotherapy protocol. The overall survival (A), event-free survival (B), and relapse-free survival rates $(C)$ were not statistically different between patients who received the ALL-type and NY regimens $(\mathrm{N}=41)$.

nitis, pulmonary fibrosis, and kyphoscoliosis requiring supportive care. A 3-year old patient with stage IV B-LBL treated with LSA2-L2 developed Langerhans cell histiocytosis (LCH) 3 years after completion of treatment, and required additional chemotherapy. Hypothyroidism developed after radiotherapy on the neck and skull, requiring medication. A 2-year old girl whose immunophenotype was unclear and who underwent initial chemotherapy with LSA2-L2, ended chemotherapy in 1999. However, she developed dilated cardiomyopathy with adriamycin, and received heart transplantation in 2007.

Comparison of outcomes by treatment period and protocols

There was no significant difference in OS when patients were categorized based on time periods [the 1990s $(\mathrm{N}=18)$, the 2000s $(\mathrm{N}=31)$, and the 2010s $(\mathrm{N}=14)$ ] according to when they underwent chemotherapy: the $1990 \mathrm{~s}: 83.3 \pm 8.8 \%$; the 2000s: $71.0 \pm 8.2 \%$; and the 2010s: $92.9 \pm 7.4 \%(P=0.188)$. The
EFS was $72.2 \pm 10.6 \%, 54.2 \pm 13.9 \%$, and $85.1 \pm 9.7 \%$ for each treatment period mentioned above, respectively, and there was no significant difference $(P=0.318)$. Although the follow-up phase for each time period was different, patients diagnosed and treated since 2010 showed the best survival.

When survival outcomes were compared by initial treatment protocol type, the NY and ALL-type protocols were performed in 27 and 14 patients, respectively. There was no significant difference in OS with $78.6 \pm 11.1 \%$ in the ALL-type protocol group and $85.0 \pm 6.9 \%$ in the NY protocol group $(P=0.540$, Fig. $5 \mathrm{~A})$. The EFS was $78.6 \pm 11.0 \%$ and $73.5 \pm 8.6 \%$ in the ALL-type and NY protocol groups, respectively ( $P=0.871$, Fig. $5 B$ ). The RFS was $78.6 \pm 11.0 \%$ and $73.5 \pm 8.6 \%$ in the ALL-type and NY protocol groups, respectively, with no significant difference $(P=0.231$, Fig. $5 C)$. In addition, there was no statistical difference in survival between the two chemotherapy groups when analyzed according to immunophenotype. 


\section{PCRT and CNS relapse}

PCRT was conducted in 13 patients (21.3\%). Among the 13 patients who received PCRT, the number of patients with stage I, II, III, IV disease was 2, 1, 4, and 6, respectively. Most patients had T-LBL (11 patients, 84.6\%), 2 patients had B-LBL, and 1 patient had an unknown immunophenotype. One of the patients was treated with LSA2-L2, 2 with AD-COMP, 2 with the ALL-type protocol, and 8 with the NY protocol.

There were 4 patients who relapsed in the CNS among the 63 patients; 3 of the patients had B-LBL and 1 patient had T-LBL. These patients had no CNS disease at diagnosis, and 1 received PCRT. The cumulative incidence of CNS relapse with PCRT was not statistically different $(P=0.859)$.

\section{DISCUSSION}

A discussion regarding the best LBL treatment has been ongoing, and recent treatment approaches have been derived from that for pediatric ALL. From the 1970s to the 1990s, LSA2-L2 was used as the gold standard treatment for LBL through a randomized trial comparing the COMP and LSA2-L2 protocols [2, 22]. Since then, several international cooperative groups have modified the LSA2-L2 protocol. In the 2000s, the effect of intensified chemotherapy through a regimen derived from the ALL regimen was announced based on the NHL/BFM-90 trial.

In the NHL/BFM-95 study, the necessity of eliminating PCRT and local radiotherapy with a flexible change of treatment direction according to induction response, have been mentioned [1, 15]. The BFM group intensified treatment with HD-MTX while reducing exposure to anthracycline, and eliminated irradiation to sites of local disease. As a result of the many NHL/BFM studies, the treatment outcomes of LBL have improved dramatically, and PCRT has been eliminated $[1,5]$.

Recently, a modified LSA2-L2 protocol (the NY protocol) and the ALL-type protocol with a BFM backbone have been the 2 main treatment approaches for pediatric LBL. The NY protocol (CCG-1901) consists of early intensive chemotherapy, followed by less intensive pulse chemotherapy during maintenance. After 4 weeks of induction and 4 weeks of consolidation, patients undergo repeated maintenance every 3 months for 2 years (Supplementary Table 1). A modified CCG-1901 protocol was used at our center. Because thioguanine was not available in Korea, mercaptopurine was used. In addition, as Asian patients have shown lower tolerability to mercaptopurine, a reduced dose of $50 \mathrm{mg} / \mathrm{m}^{2}$ was used at our institution [23]. In addition, irradiation of 1,500 cGy for the bearing area with bulk disease was included on D1-15 in the original CCG-1901, but it was excluded at our center. Additionally, in the original CCG-1901, cranial irradiation was applied on D0-14, but this was also omitted at our center.

The NY protocol has been reported to induce improved survival in pediatric LBL [24]. However, it contains a high cumulative dose of anthracycline and cyclophosphamide, and it has been argued that toxicity should be considered. The cumulative doses of anthracyclines and cyclophosphamide in the NY protocol were $360 \mathrm{mg} / \mathrm{m}^{2}$ and 10,200 $\mathrm{mg} / \mathrm{m}^{2}$, respectively, whereas those in the ALL-type protocol were $175 \mathrm{mg} / \mathrm{m}^{2}$ and $4,200 \mathrm{mg} / \mathrm{m}^{2}$ respectively $[25,26]$. Anthracycline causes cardiotoxicity, and cyclophosphamide induces hemorrhagic cystitis, bone marrow suppression, cardiotoxicity, and pulmonary fibrosis [27].

Based on the above, the BFM-based ALL-type protocol was adopted as an effective treatment strategy. Treatment was intensified with cyclophosphamide/anthracycline during the induction and consolidation, and with additional intrathecal methotrexate. In this regimen, intensive intrathecal methotrexate was applied instead of cranial irradiation, and the cumulative dose of anthracycline or cyclophosphamide was relatively decreased.

In a recent COG-A5971 study, a randomized study was conducted comparing the survival between the standard CCG-BFM ALL regimen, with or without intensification, and the standard NHL/BFM-95 regimen, with or without intensification [9]. Localized LBL was treated with the full 2-year ALL-type protocol with the BFM backbone, and the 5 -year EFS and OS were $85 \%$ and $94 \%$, respectively $[5,17]$. There was no difference in the 5-year EFS between the study arms. In addition, neither HD-MTX nor early intensifi cation with cyclophosphamide or anthracyclines improved EFS in the setting of an already intensive ALL-type regimen in LBL [28].

In this study, we analyzed the outcomes in children and adolescents with LBL treated with various regimens; the treatment outcomes were similar between the two major treatment protocols, the ALL-type and the NY protocols. The treatment results of our study were comparable with those of other previously published trials on childhood LBL. According to a report on COG-A5971, the 5-year EFS for patients with localized LBL is $90 \%$, and the OS is $96 \%$ [9]. In the case of the NY protocol, Lee et al. [4] reported treatment results of 36 pediatric patients with LBL: the 5-year EFS and OS were $79.1 \pm 3.5 \%$ and $84.7 \pm 3.1 \%$, respectively.

Late complications and secondary malignancies in longterm survivors were not included in this data. Since the cumulative dose of anticancer drugs was high in the NY protocol, it was expected that the risk of secondary malignancy and toxicity may be relatively high. Therefore, the ALL protocol could be considered for LBL treatment in the future; further studies on long-term complications are needed.

CNS relapse is associated with a poor prognosis in LBL [9]. Historically, prophylaxis for CNS-negative LBL was performed using PCRT, and showed a 90\% EFS rate, even for patients with advanced-stage T-LBL [1]. However, PCRT is associated with neuropsychological deficits such as deficits in attention concentration, and the ability to process information sequentially, mood disturbances, short stature, and secondary malignancies $[10,13]$. In addition, if patients with 
advanced stage disease showed sufficient response to induction therapy, PCRT did not confer an additive survival benefit. For this reason, contemporary regimens have safely omitted PCRT to reduce acute and long-term toxicities $[9,15]$.

In this study, PCRT was performed in 13 patients (21.3\%). Most patients had advanced stage disease (stage III or IV, 10 patients). Among the 3 patients with CNS relapse, 1 patient underwent PCRT. Although this was a retrospective analysis, there was no statistical difference in the cumulative incidence of CNS relapse, regardless of PCRT. Recent regimens, including the NHL/BFM-95 and COG-A5971 protocols, have also used this approach and reported no difference in CNS recurrence rates compared to earlier studies where cranial irradiation was administered to all high stage patients, regardless of CNS status [15]. The results of this study, in addition to the results of past studies, once again demonstrate that PCRT can be omitted in LBL.

This study has some limitations. Because a small population of patients was included from a single center, thorough data for toxicities and late effects of each regimen were not available. Analysis of a larger number of patients and information on late effects and toxicities will be needed for proper determination of the outcomes of each protocol. Furthermore, in addition to the ALL-type or NY protocols, the types of protocols applied to patients were varied. In addition, the change in criteria for selecting a treatment strategy, and improvement of supportive management during the study period of 20 years could have affected treatment outcomes. Various treatment regimens have been applied with different indications such as treatment period and immunophenotype, which could affect the results. However, when subgroup analysis was performed, proper stratification was difficult because the number of patients in each subgroup was small. Minimal disseminated disease level in the bone marrow is known to be associated with outcomes in T-cell LBL in previous studies [29]. However, the relevant data were not available in our study for technical and insurance issues. It would be meaningful if further analysis of the outcome considering minimal disseminated disease level was performed.

In conclusion, this study showed no significant difference in treatment outcomes between the ALL-type and NY protocols, regardless of immunophenotype. Furthermore, there was no difference in the incidence of CNS relapse based on PCRT. For pediatric LBL, optimized chemotherapy considering long-term complications is necessary for the future.

\section{Authors' Disclosures of Potential Conflicts of Interest}

No potential conflicts of interest relevant to this article were reported.

\section{REFERENCES}

1. Reiter A, Schrappe M, Ludwig WD, et al. Intensive ALL-type therapy without local radiotherapy provides a $90 \%$ event-free survival for children with T-cell lymphoblastic lymphoma: a BFM group report. Blood 2000;95:416-21.

2. Anderson JR, Jenkin RD, Wilson JF, et al. Long-term follow-up of patients treated with COMP or LSA2L2 therapy for childhood non-Hodgkin's lymphoma: a report of CCG-551 from the Childrens Cancer Group. J Clin Oncol 1993;11:1024-32.

3. Hvizdala EV, Berard C, Callihan T, et al. Lymphoblastic lymphoma in children--a randomized trial comparing LSA2-L2 with the A-COP+ therapeutic regimen: a Pediatric Oncology Group Study. J Clin Oncol 1988;6:26-33.

4. Lee SH, Yoo KH, Sung KW, Ko YH, Lee JW, Koo HH. Should children with non-Hodgkin lymphoma be treated with different protocols according to histopathologic subtype? Pediatr Blood Cancer 2013;60:1842-7.

5. Reiter A, Schrappe M, Parwaresch R, et al. Non-Hodgkin's lymphomas of childhood and adolescence: results of a treatment stratified for biologic subtypes and stage--a report of the BerlinFrankfurt-Münster Group. J Clin Oncol 1995;13:359-72.

6. Sekimizu M, Fujimoto J, Takimoto T, Tsurusawa M, Horibe K, Sunami S. Phase II clinical trial for newly diagnosed children and adolescents with localized lymphoblastic lymphoma (Japanese Leukemia/Lymphoma Study Group trial LLB-NHL03): study protocol for nationwide multicenter trial. Acta Med Okayama 2018;72:427-30.

7. Song JS, Youn HS, Im HJ, Ghim T, Moon HN, Seo JJ. Treatment outcome and prognostic factors for children with advanced non-Hodgkin's lymphoma at a single institution. Korean J Hematol 2006;41:157-66.

8. De Moerloose B, Suciu S, Bertrand Y, et al. Improved outcome with pulses of vincristine and corticosteroids in continuation therapy of children with average risk acute lymphoblastic leukemia (ALL) and lymphoblastic non-Hodgkin lymphoma (NHL): report of the EORTC randomized phase 3 trial 58951. Blood 2010;116:36-44.

9. Termuhlen AM, Smith LM, Perkins SL, et al. Disseminated lymphoblastic lymphoma in children and adolescents: results of the COG A5971 trial: a report from the Children's Oncology Group. Br J Haematol 2013;162:792-801.

10. Ochs J, Mulhern R, Fairclough D, et al. Comparison of neuropsychologic functioning and clinical indicators of neurotoxicity in long-term survivors of childhood leukemia given cranial radiation or parenteral methotrexate: a prospective study. J Clin Oncol 1991; 9:145-51.

11. Clayton PE, Shalet SM, Morris-Jones PH, Price DA. Growth in children treated for acute lymphoblastic leukaemia. Lancet 1988;1:460-2.

12. Schell MJ, Ochs JJ, Schriock EA, Carter M. A method of predicting adult height and obesity in long-term survivors of childhood acute lymphoblastic leukemia. J Clin Oncol 1992;10:128-33.

13. Mulhern RK, Fairclough D, Ochs J. A prospective comparison of neuropsychologic performance of children surviving leukemia who received 18-Gy, 24-Gy, or no cranial irradiation. J Clin Oncol 1991;9:1348-56.

14. Bongers ME, Francken AB, Rouwé C, Kamps WA, Postma A. Reduction of adult height in childhood acute lymphoblastic leukemia survivors after prophylactic cranial irradiation. Pediatr Blood Cancer 2005;45:139-43. 
15. Burkhardt B, Woessmann W, Zimmermann M, et al. Impact of cranial radiotherapy on central nervous system prophylaxis in children and adolescents with central nervous system-negative stage III or IV lymphoblastic lymphoma. J Clin Oncol 2006;24: 491-9.

16. Swerdlow SH, Campo E, Pileri SA, et al. The 2016 revision of the World Health Organization classification of lymphoid neoplasms. Blood 2016;127:2375-90.

17. Termuhlen AM, Smith LM, Perkins SL, et al. Outcome of newly diagnosed children and adolescents with localized lymphoblastic lymphoma treated on Children's Oncology Group trial A5971: a report from the Children's Oncology Group. Pediatr Blood Cancer 2012;59:1229-33.

18. Hoelzer D, Gökbuget N, Digel W, et al. Outcome of adult patients with T-lymphoblastic lymphoma treated according to protocols for acute lymphoblastic leukemia. Blood 2002;99:4379-85.

19. Rhee ES, Kim H, Kang SH, et al. Outcome and prognostic factors in pediatric precursor t-cell acute lymphoblastic leukemia: a single-center experience. Clin Pediatr Hematol Oncol 2018;25: 116-27.

20. Schütt P, Passon J, Ebeling P, et al. Ifosfamide, etoposide, cytarabine, and dexamethasone as salvage treatment followed by high-dose cyclophosphamide, melphalan, and etoposide with autologous peripheral blood stem cell transplantation for relapsed or refractory lymphomas. Eur J Haematol 2007;78:93-101.

21. Kobrinsky NL, Sposto R, Shah NR, et al. Outcomes of treatment of children and adolescents with recurrent non-Hodgkin's lymphoma and Hodgkin's disease with dexamethasone, etoposide, cisplatin, cytarabine, and l-asparaginase, maintenance chemotherapy, and transplantation: Children's Cancer Group Study CCG-5912. J Clin Oncol 2001;19:2390-6.
22. Anderson JR, Wilson JF, Jenkin DT, et al. Childhood nonHodgkin's lymphoma. The results of a randomized therapeutic trial comparing a 4-drug regimen (COMP) with a 10-drug regimen (LSA2-L2). N Engl J Med 1983;308:559-65.

23. Kim $H$, Seo $H$, Park $Y$, et al. APEX1 polymorphism and mercaptopurine-related early onset neutropenia in pediatric acute lymphoblastic leukemia. Cancer Res Treat 2018;50:823-34.

24. Swerdlow SH, Campo E, Harris NL, et al., eds. WHO classification of tumours of haematopoietic and lymphoid tissues. 4th ed. Lyon, France: IARC Press, 2008.

25. Büchner T, Hiddemann W, Wörmann B, Schellong G, Ritter J, Creutzig U. Acute leukemias VIII: prognostic factors and treatment strategies. Heidelberg, Germany: Springer-Verlag Berlin Heidelberg, 2001.

26. Steinherz PG, Gaynon P, Miller DR, et al. Improved disease-free survival of children with acute lymphoblastic leukemia at high risk for early relapse with the New York regimen--a new intensive therapy protocol: a report from the Childrens Cancer Study Group. J Clin Oncol 1986;4:744-52.

27. Floyd JD, Nguyen DT, Lobins RL, Bashir Q Doll DC, Perry MC. Cardiotoxicity of cancer therapy. J Clin Oncol 2005;23:7685-96.

28. Abromowitch $M$, Termuhlen A, Chang M, et al. High-dose methotrexate and early intensification of therapy do not improve 3 year EFS in children and adolescents with disseminated lymphoblastic lymphoma. Results of the randomized arms of COG A5971. Blood (ASH Annual Meeting Abstracts) 2008; 112(Suppl):3610.

29. Coustan-Smith E, Sandlund JT, Perkins SL, et al. Minimal disseminated disease in childhood T-cell lymphoblastic lymphoma: a report from the children's oncology group. J Clin Oncol 2009;27:3533-9. 